\title{
Recurrent exercise-induced rhabdomyolysis
}

\author{
Fady Hannah-Shmouni MD, Kevin McLeod MD, Sandra Sirrs MD
}

Competing interests: None declared by Fady HannahShmouni or Kevin McLeod. Sandra Sirrs has received payment for lectures from Shire Human Genetics Therapies, Genzyme Canada and Actelion Pharmaceuticals, and is an investigator for the Canadian Fabry Disease Initiative, which is funded jointly by the 10 provincial governments, Genzyme Canada and Shire Human Genetics Therapies.

This article has been peer reviewed.

\section{Correspondence to:}

Dr. Fady Hannah-Shmouni, fady.shmouni@yahoo.com

CMAJ 2012. DOI:10.1503 /cmaj.110518

1 previously healthy white 29-year-old man presented to the emergency department with a one-day history of diffuse muscle aches and dark urine following a four-hour hockey game. He had no symptoms to suggest intercurrent infection and was taking no medications. His medical history was notable for similar episodes occurring about once per year since he was 13 years old, which were never brought to medical attention. His first episode had occurred at a rigorous hockey training camp. He described triggers for muscle aches including a long delay between meals, intake of alcohol, exposure to cold temperature, sleep deprivation and prolonged intense exercise, although he was able to tolerate up to 1.5 hours of moderate exercise without symptoms. With most episodes of muscle aches, his symptoms would resolve after a short period of rest and adequate nutrition. He had no previous admissions to hospital and no exposure to anesthetics. His parents were nonconsanguineous, and there was no history of similar symptoms in his family.

When seen in the emergency department, the patient's blood pressure was 138/88 mm Hg, heart rate was 70 beats/min and regular, temperature was $36.8^{\circ} \mathrm{C}$, respiratory rate was 17 breaths/ min and his general physical examination was normal. Bulk, power and tone of muscles were normal in all muscle groups, but mild diffuse tenderness of muscles was noted on palpation. Reflexes were reduced but symmetric. He had findings suggestive of peripheral neuropathy

\section{KEY POINTS}

- The most common causes of single episodes of rhabdomyolysis are drugs, exercise and immobility.

- Defects in fatty acid $\beta$-oxidation are the most common metabolic myopathy to cause recurrent exercise-induced rhabdomyolysis.

- Some defects of fatty acid $\beta$-oxidation are common especially in Canadian Aboriginal populations, although the clinical significance of this finding is not yet clear.

- The acylcarnitine profile is a widely available and simple method used for initial screening for these defects. with decreased vibration sense in the upper and lower extremities, and decreased sensation to pinprick in the lower extremities.

Laboratory investigations were consistent with rhabdomyolysis (creatine kinase level 110190 [normal 35-250] U/L) with renal impairment (creatinine level 271 [normal 60115] $\mu \mathrm{mol} / \mathrm{L})$. Urine tested positive for myoglobin. Screening blood tests to look for acquired causes of rhabdomyolysis such as hypothyroidism, diabetic ketoacidosis, hypokalemia, hyponatremia and hypophosphatemia were normal. He was given intravenous fluids and was discharged from hospital five days later with normal renal function and creatine kinase level.

Given his history of recurrent muscle aches triggered by situations in which muscles were using fat as an energy source and physical findings of peripheral neuropathy, he was referred for evaluation of possible metabolic myopathy. Because the triggers he listed for his symptoms suggested a disorder of fatty acid $\beta$-oxidation, an acylcarnitine profile was performed, which was suggestive of long-chain 3-hydroxyacyl-coenzyme A dehydrogenase deficiency. The diagnosis was then confirmed through mutation analysis (heterozygous for the common mutation $1528 \mathrm{C}>\mathrm{G}$ ). His siblings were screened for this autosomal recessive disorder and found to be unaffected.

\section{Discussion}

We present a case of a defect in fatty acid $\beta$-oxidation in which the patient presented to the emergency department with rhabdomyolysis. Rhabdomyolysis is the breakdown of muscle tissue and leakage of its toxic content in the blood secondary to disruption of cell homeostasis. About 26000 instances of rhabdomyolysis are reported annually in the United States. ${ }^{1}$ Several ions have been implicated in the pathogenesis of this disorder with increased intracellular calcium being the final common pathway and trigger of apoptosis (cell death). ${ }^{2}$ The classic triad of presentation, muscle aches, weakness and dark urine, is seen in less than $10 \%$ of patients. ${ }^{3}$ Although first 
described in World War II victims of crush injury, rhabdomyolysis can also occur in healthy individuals following strenuous exercise. ${ }^{4}$

\section{Causes of rhabdomyolysis}

The causes of rhabdomyolysis differ in patients with single episodes versus those with recurrent disease. In a review of 97 adult patients who presented to the emergency department with single episodes of rhabdomyolysis, the most common triggers were cocaine use, exercise and immobilization. ${ }^{5}$ Other causes are outlined in Box 1. Alcohol has been implicated in up to $20 \%$ of single episodes secondary to its direct toxic effect on the muscle sarcolemma or the observation that intoxicated patients lying unconscious on a hard surface may cause direct injury to muscles from the ongoing external pressure to the tissue. ${ }^{1,4}$ It is not known why some patients develop rhabdomyolysis while others who are exposed to the same triggers do not.

\section{Acute episodes}

In an acute episode of rhabdomyolysis, clinical evaluation and simple laboratory tests will confirm the diagnosis. The most sensitive measurement for diagnosis is plasma creatine kinase level, which is usually elevated in the thousands. Serial tests for creatine kinase levels should be done every 6-12 hours, with a usual peak in 24 hours and a gradual decline thereafter. If creatine kinase levels remain elevated, compartment syndrome should be considered. ${ }^{2}$ Although nonspecific, a urine dipstick test for myoglobinuria can be indicative of the early stages of rhabdomyolysis in $50 \%$ of instances. ${ }^{2}$ More specific skeletal muscle measurements that are not readily available are carbonic anhydrase III and myosin heavy-chain fragments. ${ }^{2,3}$ Other tests that are used to diagnose complications of rhabdomyolysis such as acute kidney injury or disseminated intravascular coagulopathy include blood urea nitrogen, creatinine, platelet count, prothrombin and activated partial thromboplastin time. An interesting finding is transient hypocalcemia early in the course of the disorder (from the influx of calcium into the injured myocyte), corrected by secondary hyperparathyroidism in the later stages of disease. ${ }^{2}$

Once the diagnosis of rhabdomyolysis has been confirmed, a history of trauma, drug use and exposure to anesthetic agents should be elicited. ${ }^{2,3}$ Patients should be asked about progressive muscle weakness between episodes of rhabdomyolysis, joint inflammation and skin rash, which may be clues to the presence of inflammatory myopathies such as polymyositis. Constitutional symptoms may suggest an infec- tious etiology. Simple blood tests such as electrolytes, glucose and thyroid-stimulating hormone can be used to rule out other acquired causes of the disorder. ${ }^{2}$

\section{Acute treatment}

Guidelines on acute treatment of rhabdomyolysis indicate that supportive therapy with isotonic crystalloids helps to maintain renal function, with a goal of urine output of $200-300 \mathrm{~mL} / \mathrm{h}$. ${ }^{6}$ The guidelines also address avoidance of triggers, correction of electrolyte abnormalities, such as hyperkalemia, and various diuretics (e.g., mannitol) as well as bicarbonate therapy to alkalinize urine for the prevention of myoglobin cast precipitation in the kidney tubules. ${ }^{6}$

\section{Recurrent episodes}

All patients with recurrent rhabdomyolysis should undergo investigation for an underlying cause, although in many of these patients, no cause will be identified. Recurrent rhabdomyolysis may be caused by acquired myopathies such as polymyositis, congenital muscle disorders such as muscular dystrophy ${ }^{7}$ or by metabolic myopathies. There are three main groups of metabolic myopathies: disorders of fat metabolism, disorders of carbohydrate metabolism and disorders of mitochondrial function. ${ }^{8,9}$ Table 1 describes features on history and physical examination that can be used to distinguish these three broad classes of metabolic myopathies.

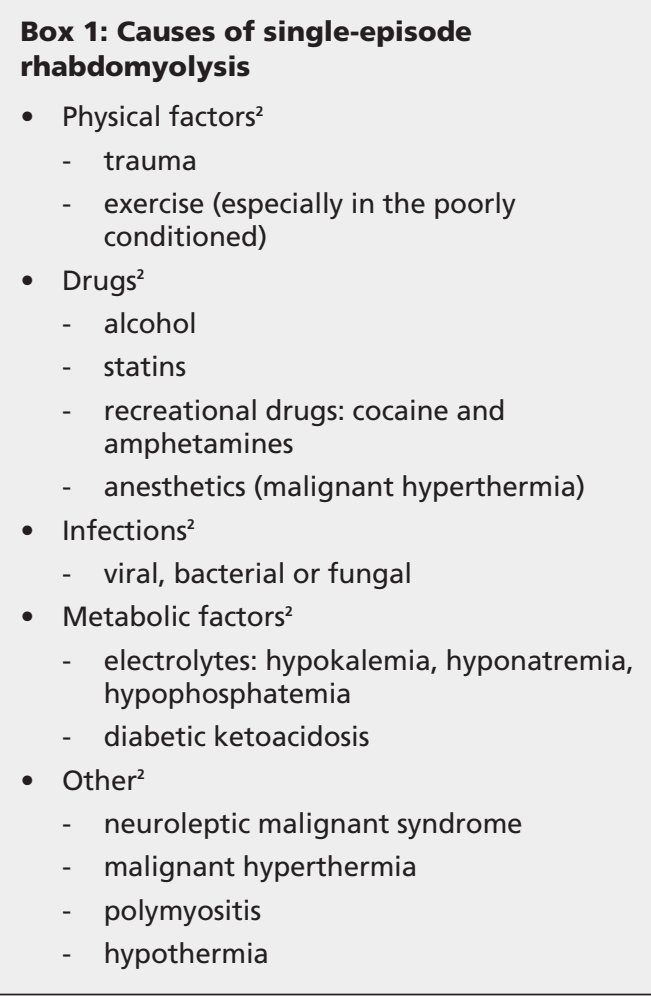


Simple points on history such as duration of exercise needed to bring on symptoms, secondwind phenomenon and the presence of symptoms in other organ systems will help clinicians decide which of the three main groups of metabolic myopathies (disorders of fatty acid $\beta$-oxidation, muscle glycogenoses and mitochondrial diseases) should be considered. Also, a family history of consanguinity or sudden infant death syndrome may be suggestive of a metabolic cause.

When patients are presenting with symptoms of a disorder of fatty acid $\beta$-oxidation, creatine kinase levels are usually elevated (> $100000 \mathrm{U} / \mathrm{L}$ ) and then return to normal when energy demand is met. It is this normalization of creatine kinase levels between episodes that is a clue to the presence of a disorder of fatty acid $\beta$-oxidation, as creatine kinase levels remain elevated in other causes of muscle pain such as inflammatory myopathy and muscle glycogenosis. ${ }^{89}$

It is important to identify patients with a possi- ble disorder of fatty acid $\beta$-oxidation with clues on history and physical examination, because this diagnosis can be confirmed with blood testing, whereas the other causes of metabolic myopathies often require muscle biopsy for confirmation.

\section{Disorders of fatty acid $\beta$-oxidation}

The most common cause of recurrent exerciseinduced rhabdomyolysis is a disorder of fatty acid $\beta$-oxidation, ${ }^{8,9}$ and the pathway of fatty acid $\beta$ oxidation is summarized in Figure 1. In patients with disorders of fatty acid $\beta$-oxidation, provision of energy from fat is not sufficient to cover demand, especially for organs such as the heart and skeletal muscles with high energy requirements during exposure to cold, infection or fasting. ${ }^{10}$ The ability to perform short-duration, intense exercise is not impaired in people with disorders of fatty acid $\beta$-oxidation because glycogen is the primary substrate used for energy production during the first 10-30 minutes of exercise. ${ }^{89}$

Table 1: Metabolic myopathies causing recurrent-episode rhabdomyolysis

\begin{tabular}{|c|c|c|c|}
\hline Characteristic & Disorders of fatty acid $\beta$-oxidation & Muscle glycogenoses & Mitochondrial diseases \\
\hline \multirow{2}{*}{ Prevalence } & MCAD 1/10 000 & McArdle disease $1 / 100000^{8,9}$ & \\
\hline & $\begin{array}{l}\text { Others less common except in } \\
\text { certain populations }\end{array}$ & Others less common & \\
\hline Inheritance pattern & Most are autosomal recessive $\mathrm{e}^{8,9}$ & $\begin{array}{l}\text { Most are autosomal } \\
\text { recessive }^{8,9}\end{array}$ & $\begin{array}{l}\text { Any pattern of inheritance is } \\
\text { possible: maternally } \\
\text { inherited through } \\
\text { mitochondrial DNA or } \\
\text { inherited through nuclear } \\
\text { DNA in autosomal dominant } \\
\text { or recessive or X-linked } \\
\text { fashion }^{8,9}\end{array}$ \\
\hline $\begin{array}{l}\text { Duration of exercise needed } \\
\text { to trigger symptoms }\end{array}$ & Longer duration $(>30 \mathrm{~min})^{8,9}$ & Shorter duration $(<30 \mathrm{~min})^{8,9}$ & Variable $e^{8,9}$ \\
\hline Other organ systems involved & $\begin{array}{l}\text { Depends on type } \\
\text { Adult often present only with } \\
\text { rhabdomyolysis } \\
\text { Other symptoms may include } \\
\text { encephalopathy, cardiomyopathy, } \\
\text { peripheral neuropathy or } \\
\text { hypoketotic hypoglycemia }\end{array}$ & $\begin{array}{l}\text { Depends on type } \\
\text { May have hepatomegaly and } \\
\text { history of hypoglycemia; } \\
\text { some types have cardiac } \\
\text { involvement }^{8,9}\end{array}$ & $\begin{array}{l}\text { Other organ systems } \\
\text { frequently involved, with } \\
\text { cardiac, endocrine and } \\
\text { central nervous systemic } \\
\text { involvement common }\end{array}$ \\
\hline $\begin{array}{l}\text { Investigations leading to } \\
\text { diagnosis }\end{array}$ & $\begin{array}{l}\text { Acylcarnitine profile will suggest } \\
\text { diagnosis, which can be } \\
\text { confirmed by mutation analysis or } \\
\text { fibroblast culture to assess specific } \\
\text { enzymes in fatty acid } \beta \text {-oxidation } \\
\text { pathway }{ }^{8,9}\end{array}$ & $\begin{array}{l}\text { Diagnosis often found on } \\
\text { muscle biopsy showing } \\
\text { increased glycogen stores; } \\
\text { subtyping of the type of } \\
\text { glycogen storage disease may } \\
\text { require further enzyme or } \\
\text { DNA analysis } \text { s.9 }^{8,9}\end{array}$ & $\begin{array}{l}\text { Muscle biopsy with analysis } \\
\text { for defects in mitochondrial } \\
\text { DNA or nuclear DNA }\end{array}$ \\
\hline
\end{tabular}

MCAD = medium-chain acyl-coenzyme A dehydrogenase.

*Second-wind phenomenon: improvement in exercise tolerance 10-30 minutes into exercise as muscle metabolism switches from using carbohydrates as a source of energy to using fat. 
Three disorders of fatty acid $\beta$-oxidation are particularly common: medium-chain acylcoenzyme A dehydrogenase (MCAD) deficiency and carnitine palmitoyltransferase II (CPT2) deficiency, which are pan-ethnic, ${ }^{8,9}$ and carnitine palmitoyltransferase I (CPT1) deficiency in Canadian Aboriginal populations.

Medium-chain acyl-coenzyme A dehydrogenase deficiency, one of the inborn errors of metabolism that can present with sudden infant death syndrome, was the first disorder of fatty acid $\beta$-oxidation to be added to newborn screening panels around the world. ${ }^{11,12}$ Once newborn screening for MCAD was in place, data were published showing that the prevalence of MCAD deficiency of roughly $1 / 10000$ ascertained through newborn screening was three to four times higher than that ascertained through clinical presentation. ${ }^{11,12}$ This discrepancy suggests that a large number of patients with MCAD deficiency may not receive diagnoses in childhood. A recent publication that reviewed instances of MCAD deficiency diagnosed in adults found that rhabdomyolysis can be the presenting symptom; other presentations included encephalopathy, cardiomyopathy and hypoglycemia. ${ }^{12}$ The mortality rate of these newly diagnosed instances of MCAD deficiency in adults was high (29\%). ${ }^{12}$ Thus, it is clear that the first presentation of a disorder of fatty acid $\beta$-oxidation may occur in adult patients.

Carnitine palmitoyltransferase II deficiency is the most common metabolic myopathy presenting with rhabdomyolysis in adults. ${ }^{89}$ These patients primarily present with myopathic symptoms: muscle aches, cramps, muscle stiffness and weakness, beginning in the first or second decade of life. The severity of attacks range from mild muscle aches to life-threatening rhabdomyolysis. The triggers include situations in which lipid metabolism is used for energy production as seen in illness, fasting, diet with high intake of fat, drugs (e.g., ibuprofen, acetaminophen, anesthetics) and prolonged exercise, ${ }^{8,9}$ as in our patient.

A third gene defect in disorders of fatty acid $\beta$-oxidation involving CPT1 has recently been shown to be very common in Canadian Aboriginal populations. A recent public symposium in British Columbia on disorders of fatty acid $\beta$ oxidation in First Nations showed evidence that up to $25 \%$ of the Canadian Aboriginal population living on Vancouver Island are homozygous for a mutation in CPT1 (P479L), which is also highly prevalent in other Canadian Aboriginal populations, including those in some regions of Nunavut. ${ }^{13,14}$ The prevalence of this defect is related to infant death in one study from Alaska. ${ }^{15}$ It is not yet clear if adults with this disorder are at risk of symptoms, so further data are needed to define the clinical impact of the CPT1 mutation in the Canadian Aboriginal population.

If a clinician suspects that rhabdomyolysis is caused by a disorder of fatty acid $\beta$-oxidation, investigations should begin with testing of fasting blood glucose and lactate levels, as well as a simple screening test known as the acylcarnitine profile. ${ }^{8.9}$ The profile is measured using tandem mass spectrometry and can identify more than 20 different metabolic defects of fatty acid and organic acid metabolism. ${ }^{8,9}$ The acylcarnitine profile can be measured in a bloodspot (in which a small amount of blood is spotted into filter paper, similar to the technique used to perform screening in newborns) or measured in serum. ${ }^{8,9}$ This test can be done at all laboratories that perform screening in newborns, and therefore clinicians everywhere in the country can access this simple and inexpensive test. The diagnosis is confirmed with mutation analysis and fibroblast cultures of enzyme activity. ${ }^{89}$ Mitochondrial and glycogen storage diseases require a muscle biopsy for diagnosis. ${ }^{8,9}$

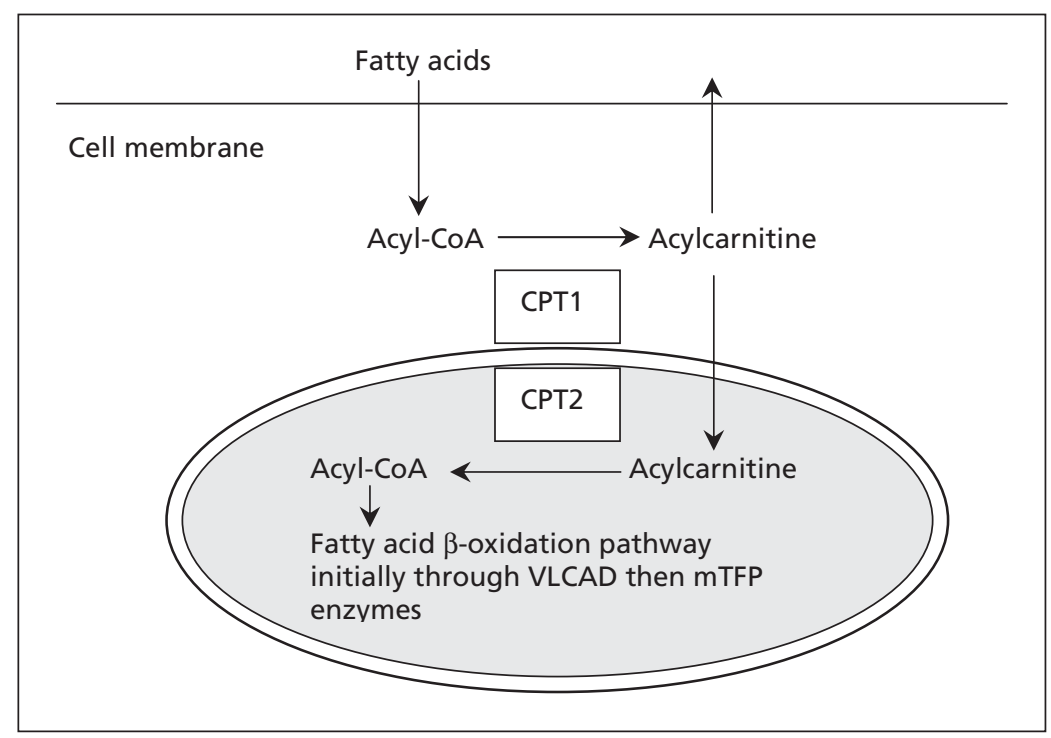

Figure 1: Simplified illustration of fatty acid $\beta$-oxidation. Fatty acids enter cells through specialized receptors. Once in the cytoplasm, fatty acids are activated through esterification (i.e., joined to coenzyme A [CoA] to form acyl-CoA). The acyl-CoA is then linked to a carnitine molecule and this acylcarnitine is shuttled by two enzymes (CPT1 and CPT2) through the outer and inner mitochondrial membranes. The carnitine molecule is removed, leaving the acyl-CoA, which is metabolized by a series of enzymes including very-long-chain acyl-CoA dehydrogenase (VLCAD) and mitochondrial trifunctional protein (mTFP) that consists of hydratase, long-chain 3-hydroxylacyl CoA dehydrogenase (LCHAD) and thiolase activity, into smaller fatty acid chains, which are ultimately converted into adenosine triphosphate (ATP) within the mitochondrion. In our patient, deficiency of LCHAD lead to a backup of long-chain acyl-CoA species that could not be metabolized, and the resultant deficiency of ATP lead to rhabdomyolysis. These long-chain species are converted to acylcarnitine, which is readily permeable to cross the cell membrane and can be detected with a simple blood test. For details about the biochemical pathways of fatty acid $\beta$-oxidation, see The Online Metabolic and Molecular Bases of Inherited Disease ${ }^{8}$ and Online Mendelian Inheritance in Man. ${ }^{9}$ CPT = carnitine palmitoyltransferase. 


\section{Prevention of recurrent episodes}

All patients with recurrent episodes of rhabdomyolysis, regardless of cause, should be taught to avoid triggers and ensure adequate hydration, and encouraged to warm up before exercise. Patients with disorders of fatty acid $\beta$ oxidation can also adopt a low-fat diet to reduce the frequency of rhabdomyolysis. ${ }^{16}$ Other modalities of treatment for disorders of fatty acid $\beta$ oxidation include replacement of essential fatty acids in the diet using supplemental walnut or soy oils. Carnitine supplementation is frequently used, although there is no evidence that this will affect outcome. ${ }^{17}$ Although these therapies for disorders of fatty acid $\beta$-oxidation may reduce the frequency of episodes of rhabdomyolysis, other long-term complications, such as cardiomyopathy, retinopathy and neuropathy, continue to occur. ${ }^{16} \mathrm{~A}$ recent trial of bezafibrate was shown to restore the capacity for normal fatty acid $\beta$-oxidation in muscle cells from patients with a mild form of CPT2 deficiency, an approach which may be useful for other disorders of fatty acid $\beta$-oxidation. ${ }^{18}$

\section{References}

1. Graves EJ, Gillum BS. Detailed diagnoses and procedures, National Hospital Discharge Survey, 1995. Vital Health Stat 13 1997; 13:1-146

2. Sauret O, Marinides G, Wang G. Rhabdomyolysis. American Fam Physician 2002;65:907-12

3. Bosch X, Poch E, Grau JM. Rhabdomyolysis and acute kidney injury. N Engl J Med 2009;361:62-72.

4. Sinert R, Kohl L, Rainone T, et al. Exercise-induced rhabdomyolysis. Ann Emerg Med 1994;23:1301-6.

5. Fernandez WG, Hung O, Bruno GR, et al. Factors predictive of acute renal failure and need for hemodialysis among ED patients with rhabdomyolysis. Am J Emerg Med 2005;23:1-7.

6. Finnish Medical Society Duodecim. Rhabdomyolysis. In: Evidence-based medicine guidelines. Helsinki (Finland): Wiley Interscience, John Wiley \& Sons; 2007.

7. Miller ED Jr, Sander DB, Rowlingson JC, et al. Anesthesiainduced rhabdomyolysis in a patient with Duchenne's muscular dystrophy. Anesthesiology 1978;48:146-8.

8. Saudubray J-M, Charpentier C. Chapter 66: clinical phenotypes: diagnosis/algorithms. The online metabolic and molecular bases of inherited disease. Columbus $(\mathrm{OH})$ : The McGraw-Hill Companies. Available: www.ommbid.com/OMMBID/the_online_metabolic _and_molecular_bases_of_inherited_disease/b/abstract/Part6/ch66 (accessed 2011 Dec. 15).

9. Acyl-CoA dehydrogenase, medium-chain; ACADM. Online Mendelian inheritance in man. Baltimore (MD): Johns Hopkins University. Available: http://omim.org/entry/607008 (accessed 2011 Dec. 15).

10. Spiekerkoetter U. Mitochondrial fatty acid oxidation disorders: clinical presentation of long-chain fatty acid oxidation defects before and after newborn screening. J Inherit Metab Dis 2010;33: 527-32.

11. Derks TG, Duran M, Waterham HR, et al. The difference between observed and expected prevalence of MCAD deficiency in the Netherlands: a genetic epidemiological study. Eur J Hum Genet 2005; 13:947-52.

12. Lang TF. Adult presentations of medium-chain acyl-CoA dehydrogenase deficiency (MCADD). J Inherit Metab Dis 2009;32:675-83.

13. Sinclair G, Ma J, MacLeod P, et al. Retrospective genotyping of newborn screening cards for the P479L carnitine palmitoyltransferase (CPT1) variant: correlation with acylcarnitine profiles and estimation of incidence in British Columbia [abstract]. Mol Genet Metab 2007;90:262.

14. Greenberg CR, Dilling LA, Thomposon GR, et al. The paradox of the CPT1a P479L variant in Canadian Aboriginal populations. Mol Genet Metab 2009;96:201-7.

15. Gessner BD, Gillingham MB, Birch S, et al. Evidence for an association between infant mortality and a carnitine palmitoyltransferase 1A genetic variant. Pediatrics 2010;126:945-51.

16. Spiekerkoetter U, Linder M, Santer R, et al. Management and outcome in 75 individuals with long-chain fatty acid oxidation defects: results from a workshop. J Inherit Metab Dis 2009;32: 488-97.

17. Winter SC. Treatment of carnitine deficiency. J Inherit Metab Dis 2003;26:171-80

18. Bonnefont JP, Bastin J, Behin A. Bezafibrate for an inborn mitochondrial beta oxidation defect. N Engl J Med 2009;360:838-40.

Affiliations: From the Adult Metabolic Diseases Clinic, Division of Endocrinology, University of British Columbia, Vancouver, BC

Contributors: Each author contributed equally to the conception, design, analysis, data collection, interpretation, writing, critical revision and final approval of the article. Sandra Sirrs guarantees the scientific integrity of the work as a whole.

Acknowledgement: The authors thank Dr. Graham Sinclair for performing the acylcarnitine analysis.

The section Cases presents brief case reports that convey clear, practical lessons. Preference is given to common presentations of important rare conditions, and important unusual presentations of common problems. Articles start with a case presentation (500 words maximum), and a discussion of the underlying condition follows (1000 words maximum). Visual elements (e.g., tables of the differential diagnosis, clinical features or diagnostic approach) are encouraged. Written consent from patients for publication of their story is a necessity and should accompany submissions. See information for authors at www.cmaj.ca.

CMAJ remains committed to notifying readers in a timely way about advisories and warnings pertaining to serious adverse drug events. A collection of recent drug advisories from Health Canada and the US Food and Drug Administration is regularly updated at www.cmaj.ca/misc/advisories.xhtml. 